Recebido em 03/2020. Aceito para publicação em 09/2020.

\title{
IDENTIFICAÇÃO E ANÁLISE DE EVENTOS HILDCAA/HILDCAA* PARA O ANO DE 1998 USANDO PYTHON
}

\section{IDENTIFICATION AND ANALYSIS OF EVENTS HILDCAA/HILDCAA* FOR THE YEAR 1998 USING PYTHON}

\author{
Isabelle Cristine Pellegrini Lamin ${ }^{1}$ \\ Virgínia Klausner de Oliveira ${ }^{2}$ \\ Arian Ojeda González ${ }^{3}$ \\ Alan Prestes 4 \\ Valdir Gil Pillat ${ }^{5}$ \\ Marina Cezarini ${ }^{6}$
}

Resumo: O artigo visa o desenvolvimento de um novo algoritmo via Python para estudar eventos de Atividade Auroral Contínua do Índice AE (Eletrojato Auroral), de Grande Intensidade e Longa Duração, HILDCAAs e HILDCAAs* (no qual * corresponde ao fenômeno HILDCAA flexibilizado). Este algoritmo é baseado e validado por meio do algoritmo previamente desenvolvido por Prestes et al. (2017a) em MATLAB. O intuito deste novo algoritmo proposto aqui é tornar o fluxograma deste acessível a todos os usuários, além de complementar e atualizar o algoritmo em MATLAB já existente. Um importante aspecto é o fato da linguagem de programação de alto nível Python ser uma ferramenta gratuita. Ademais, o artigo também objetiva a comparação dos eventos encontrados no ano de 1998 neste trabalho com aqueles obtidos por Prestes et al. (2017a) e Guarnieri (2006). A nova flexibilização adotada aqui não tem a intensão de suprimir ou modificar a concepção original das HILDCAAs, mostra que os eventos encontrados aqui continuam associados aos fenômenos HSSs/CIRs (Feixes Rápidos do Vento Solar/Regiões de Interações Corrotantes).

Palavras-chave: HILDCAA; Python; CIRs; HSSs.

\begin{abstract}
The project aims to study High Intensity and Long Duration, Continuous AE (Geomagnetic Auroral Eletroject index) Auroral Activity events (HILDCAAs) and HILDCAAs* (in which * corresponds to a less strict HILDCAA phenomenon). This algorithm is based on and validated using the code previously developed by Prestes et al. (2017a) in MATLAB. The purpose of the new algorithm proposed here is to make the code flow chart accessible to all users, in addition to complementing and updating the existing program in MATLAB. Another important aspect is the fact that the high-level programming language Python is a free source. Moreover, the paper also aims to compare the events found in the year 1998 obtained using the new code developed in Python with those by Prestes et al. (2017a) and Guarnieri (2006). The relaxation adopted here does not intend to suppress or modify the original conception on HILDCAAs, and shows that our events are still associated with the HSSs/CIRs (High Speed Streams/ Corotating Interaction Regions) events.
\end{abstract}

Keywords: HILDCAA, Python, CIRs, HSSs.

\section{INTRODUÇÃO}

A geofísica tem por objetivo descrever os fenômenos físicos do planeta Terra,

\footnotetext{
1 Universidade do Vale do Paraíba - Univap, Brasil. E-mail: isa_lamin@hotmail.com.

2 Universidade do Vale do Paraíba - Univap, Brasil. E-mail: viklausner@gmail.com.

3 Universidade do Vale do Paraíba - Univap, Brasil. E-mail: ojeda.gonzalez.a@gmail.com

${ }^{4}$ Universidade do Vale do Paraíba - Univap, Brasil. E-mail: aprestes@gmail.com.

5 Universidade do Vale do Paraíba - Univap, Brasil. E-mail: valdirgp@univap.br.

${ }^{6}$ Universidade do Vale do Paraíba - Univap, Brasil. E-mail: marina.cezarini@gmail.com.
} 
como por exemplo a gravimetria, aeronomia, sismologia, geotermia, entre outras. Este trabalho está inserido na aeronomia, a qual estuda as características físicas e químicas da alta atmosfera, e a qual depende diretamente das variações da atividade solar e do campo magnético. O planeta Terra está sujeito às radiações, partículas, e campos magnéticos emanadas do Sol, entretanto, o campo magnético terrestre desempenha o papel de "camada protetora". Portanto, a magnetosfera é a região que "isola" o planeta Terra dos fenômenos do meio interplanetário, como por exemplo, do vento solar. Em outras palavras, a magnetosfera atua como um escudo protetor possibilitando a existência de vida, e permite que seres vivos habitem o planeta Terra (RATCLIFFE, 1972).

Nesse sentido, as variações da atividade solar são responsáveis pelo fluxo de partículas dentro da magnetosfera. Em decorrências destas atividades, as variações de regime estacionário do vento solar, principalmente variações de densidade e temperatura aliadas ao campo magnético ocasionam distúrbios na magnetosfera. Como exemplo prático deste tipo de interação vento solar/magnetosfera, pode-se mencionar um fenômeno muito conhecido devido a penetração de partículas energéticas atingindo altitudes atmosféricas, as conhecidas auroras boreal e austral (CHAPMAN; FERRARO, 1930; 1931; 1933).

Neste contexto, existem dois tipos de tempestades geomagnéticas: (1) tempestade geradas por Ejeção de Massa Coronal (CME) e (2) tempestade geradas por HSS/CIR. A segunda está relaciona a presença de buracos coronais no Sol. Estes buracos dão origem a HSSs, e, consequentemente, a CIRs. A interação entre a magnetosfera terrestre e os HSSs/CIRs ocasiona distúrbios da atividade geomagnética pois altera os sistemas de correntes nas regiões polares, mensurados pelo índice eletrojato auroral $(\mathrm{AE})$, que será discutido a seguir.

Um dos eventos relacionados a HSSs/CIRs são as HILDCAAs, Atividade Auroral Contínua do índice AE (do inglês Auroral Electrojet), de Grande Intensidade e Longa Duração (do inglês High-Intensity Long-Duration Continuous Auroral Activity). Esses eventos HILDCAAs têm sido estudados por diversos autores (TSURUTANI; GONZALEZ, 1987; GONZALEZ et al., 1994; HAJRA et al., 2014; HAJRA et al., 2015; SOUZA et al., 2016; PRESTES; KLAUSNER; GONZALEZ, 2017).

Durante eventos HILDCAAs, com as variações dos parâmetros do vento solar há uma intensificação nas correntes ovais aurorais, que resultam em distúrbios nos valores de campo geomagnético em diversas longitudes localizadas em regiões de altas latitudes do hemisfério Norte e Sul quantificados pelo índice AE. Este é um índice magnético de alta resolução temporal, ou seja, obtido a cada minuto. É obtido a partir do monitoramento das perturbações geomagnéticas medidas por no mínimo 10 observatórios geomagnéticos localizados em regiões sub-aurorais e aurorais no Hemisfério Norte (SOUZA et al., 2016; TSURUTANI; GONZALEZ, 1987; GONZALEZ et al., 1989). 
Para o auxílio da interpretação da atividade geomagnética durante a ocorrência de eventos HILDCAAs, utiliza-se o índice Dst (do inglês Disturbance Storm Time). Este índice é obtido pela média da componente horizontal do campo magnético medida em observatórios localizados em baixas e médias latitudes, entre os principais observatórios utilizados pode-se mencionar: Kakioka (KAK), Honolulu (HON), Hermanus (HER) e San Juan (SJG). O índice Dst mede a intensidade da corrente de anel, entretanto, cada observatório magnético apresenta suas particularidades locais (Klausner et al., 2013; 2014; 2015; 2016a; 2016b).

De acordo com Tsurutani e Gonzalez (1987), HILDCAAs são definidas pelos seguintes critérios:

1) A duração do evento deve ser superior ou igual a dois dias;

2) Os valores de pico do índice AE devem exceder $1.000 \mathrm{nT}$ pelo menos uma vez durante a duração do evento;

3) O índice AE não pode registrar valores menores que $200 \mathrm{nT}$ durante intervalos maiores que duas horas consecutivas;

4) Os eventos devem ocorrer fora da fase principal de tempestades geomagnéticas, visto que nesta fase da tempestade geomagnética há uma intensificação da Corrente de Anel que deve ser desconsiderada como parte do evento.

Utilizando os critérios acima mencionados, Guarnieri (2006) identificou e estudou os eventos HILDCAAs ocorridos no período de 1998 a 2001. Além disso, para verificar se o evento HILDCAA se encontrava fora da fase principal da tempestade geomagnética, Guarnieri (2006) considerava o início do evento a partir do menor valor detectado pelo índice Dst, caracterizando assim o começo da fase de recuperação, e por conseguinte, o início do evento HILDCAA. Reanalisando os critérios estabelecidos para os eventos HILDCAAs, Prestes et al. (2017) consideraram que poderia realizar uma flexibilização quanto ao terceiro critério estabelecido por Tsurutani e Gonzalez (1987), definindo estes "novos" eventos como HILDCAAs*. Ao invés do índice AE não registrar valores menores que $200 \mathrm{nT}$ durante períodos maiores que duas horas de cada vez, em HILDCAAs* estes intervalos se estenderiam para quatro horas consecutivas. Este estudo verificou que os eventos HILDCAAs* possuíam características similares aos eventos HILDCAAs, sendo estas relacionadas principalmente aos seus forçantes, isto é, aos mesmos fenômenos do meio interplanetário. Vale ressaltar que para a identificação de HILDCAAs, Prestes et al. (2017) consideraram os critérios definidos por Tsurutani e Gonzalez (1987). E para atender ao quarto critério, consideravam que os eventos HILDCAAs estavam fora da fase principal da tempestade magnética somente quando a fase de recuperação iniciava e atingia Dst>-50 nT.

Dando continuidade aos estudos realizados por Guarnieri (2006), e considerando os critérios e características dos eventos HILDCAAs/HILDCAAs*, Prestes et al. (2017) desenvolveram um algoritmo via MATLAB para a detecção dos eventos no decorrer dos 
anos de 1998 a 2007. Pode-se citar que o algoritmo foi divulgado na comunidade científica pela primeira vez por Prestes et al. (2017). Neste trabalho, foi descrito o passo a passo do algoritmo desenvolvido, em conjunto com o fluxograma do mesmo. Essa ferramenta contribuiu ainda para a análise estatística dos eventos, e para correlacionálos com os eventos HSSs/CIRs (GUARNIERI, 2006; HAJRA et al., 2014; HAJRA et al., 2015). Sendo assim, Prestes et al. (2017) contabilizaram a quantidade de eventos no período decorrente entre 1998 e 2007, e redigiram uma lista com os todos eventos encontrados (data, hora e minuto de início e fim) e o seus respectivos forçantes interplanetários associados.

Lamin et al. (2017) executaram o algoritmo via MATLAB citado acima, e detectaram que havia candidatos aos eventos HILDCAA/HILDCAA* descartados por Prestes et al. (2017) devido ao rigor do seu quarto critério. Ou seja, Lamin et al. (2017) encontraram mais eventos, porém estes ocorriam dentro da fase principal da tempestade geomagnética, sendo considerados falsos positivos e denominados "HILDCAAs*?". Os eventos "HILDCAAs*?" apresentaram um comportamento duvidoso devido à flutuação do índice Dst e à complexidade da tempestade geomagnética, e por esse motivo, gerou controvérsias quanto aos resultados dos pesquisadores supracitados. Afim de ilustrar as diferenças em definição de HILDCAA, HILDCCA*, HILDCAA*?, a Tabela 1 mostra detalhadamente como cada critério é definido.

Tabela 1 - Critérios de definições.

\begin{tabular}{lccc}
\hline \multicolumn{1}{c}{ Critério } & HILDCAA & HILDCAA $^{*}$ & HILDCAA*? $^{*}$ \\
\hline 1)Longa Duração & $>=2$ dias & $>=2$ dias & $>=2$ dias \\
2)Alta Intensidade & $1.000 \mathrm{nT}$ & $1.000 \mathrm{nT}$ & $1.000 \mathrm{nT}$ \\
3) Atividade Contínua do AE & 2 horas & 4 horas & Noras \\
$\begin{array}{l}\text { 4)Fora da Fase Principal da } \\
\text { Tempestade Magnética }\end{array}$ & Sim & Sim & Não necessariamente \\
\hline
\end{tabular}

Fonte: Os autores.

Portanto, este artigo tem como propósito a efetivação do algoritmo em Python, listando os eventos HILDCAAs/HILDCAAs* recorrentes no ano de 1998. Os resultados encontrados serão comparados com os encontrados por Guarnieri (2006) e Prestes et al. (2017). Ademais, esta ferramenta irá possibilitar a verificação da ocorrência dos eventos falsos positivos, denominados HILDCAAs*?, e analisar se estes poderão ser inclusos nas listas de eventos HILDCAAs.

\section{METODOLOGIA}

Com base no algoritmo elaborado por Prestes et al. (2017), foi desenvolvido um algoritmo para identificação de eventos HILDCAAs em Python pelo grupo do Laboratório 
de Imagens e Sinais (LIS/UNIVAP) o qual os autores integram. Além disso, nosso algoritmo objetiva a validação dos resultados obtidos pelo algoritmo via MATLAB de Prestes et al. (2017).

A Figura 1 mostra o fluxograma do algoritmo de Prestes et al. (2017) para identificação de HILDCAAs. Com base neste fluxograma, foi desenvolvido neste artigo o algoritmo em Python (veja Figura 2), para a detecção de eventos HILDCAA e HILDCAA* $^{*}$ Os fluxogramas ilustrados nas Figuras 1 e 2 mostram de modo didático a diferença entre os algoritmos desenvolvidos em MATLAB e em Python.

Figura 1 - Fluxograma de deteç̧ão de HILDCAA/HILDCAA* desenvolvido por Prestes et al. 2017a em MATLAB.

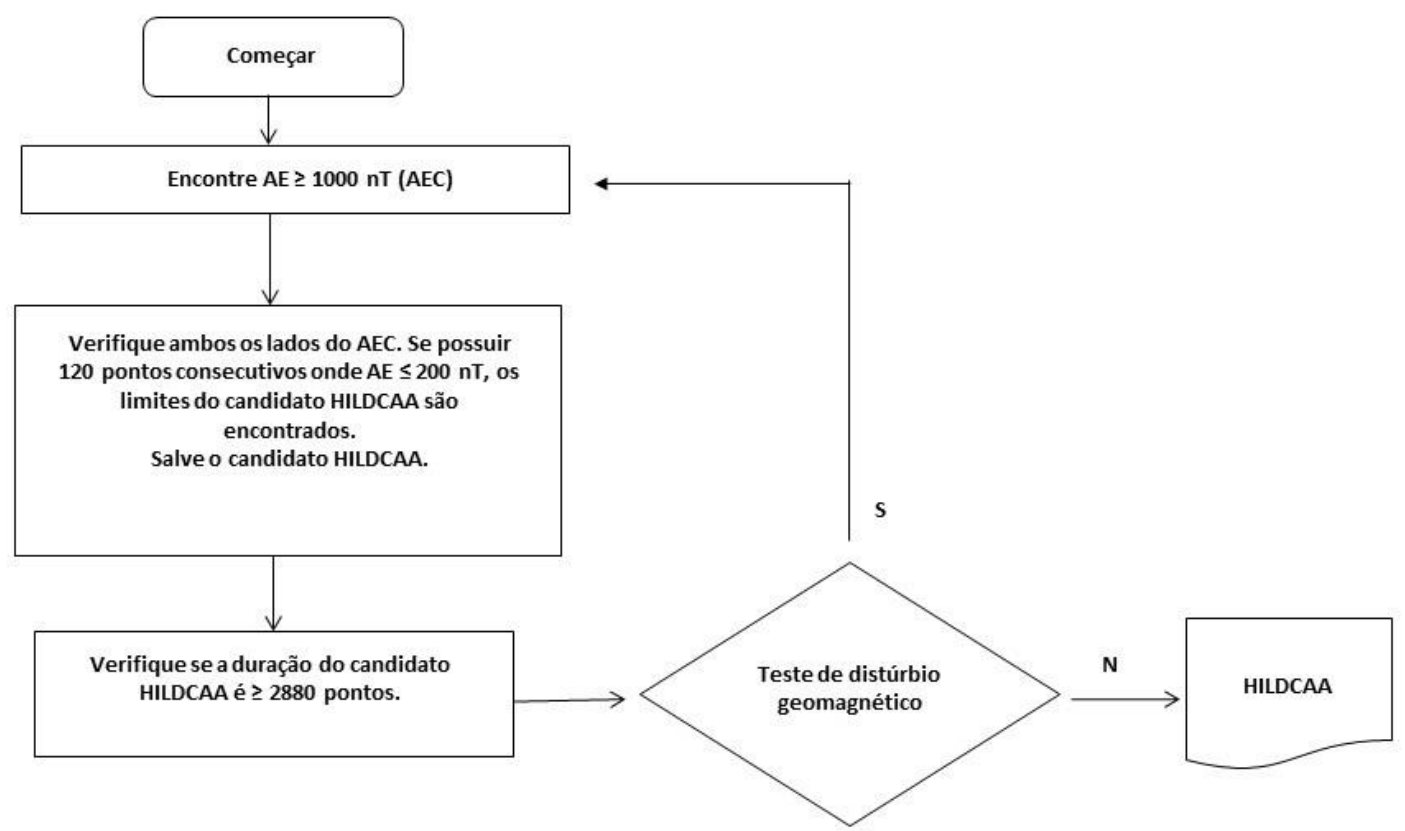

Fonte: Adaptado de Prestes et al., (2017). 
Figura 2 - Fluxograma do algoritmo desenvolvido em Python para detecção de HILDCAA/HILDCAA*.

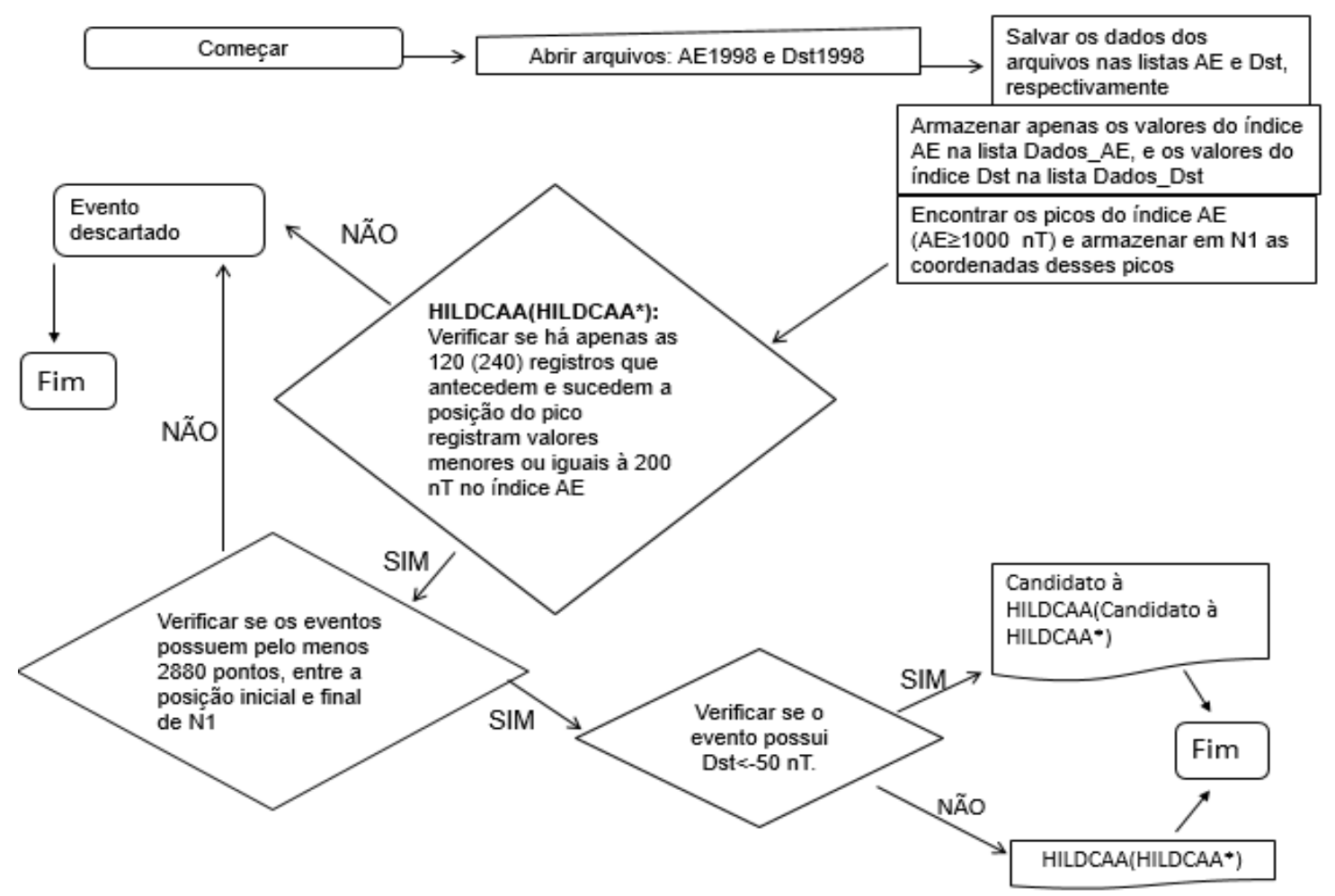

Fonte: Os autores.

O algoritmo em Python será aplicado para os eventos ocorridos no ano de 1998, além disso, estes eventos serão comparados com os eventos encontrados por Prestes et al. (2017) e Guarnieri (2006). A seguir, uma breve descrição do mesmo será dada.

Inicialmente, o algoritmo abre dois arquivos, um com os valores do índice $A E$ e outro com registros do índice SYM-H referente ao ano analisado (http://wdc.kugi.kyotou.ac.jp/index.html). Apesar do algoritmo utilizar o índice SYM-H ao invés do índice Dst, isto não é um problema pois o índice SYM-H é semelhante ao índice Dst, mas com resolução temporal de 1 min.

Posteriormente, o algoritmo deve executar os critérios que definem HILDCAAs conforme estabelecido por Tsurutani e Gonzalez (1987). O primeiro passo é criar um laço para encontrar os picos do índice AE, ou seja, valores maiores ou iguais a 1.000 $\mathrm{nT}$, atendendo ao critério (2). Uma nova lista deve ser criada para guardar essas posições de picos AE maiores ou iguais a $1.000 \mathrm{nT}$ representado por 'N1' no código, porque por meio destas coordenadas que se estabelece a duração de um possível candidato à HILDCAA ou evento HILDCAA, posteriormente.

De acordo com o critério (3), os valores de AE não podem apresentar valores menores do que $200 \mathrm{nT}$ por períodos superiores a duas horas. Dessa forma, utiliza-se a última posição do pico AE maior ou igual a $200 \mathrm{nT}$ para constar se há 120 pontos 
consecutivos (dados com resolução de minutos, i.e., duas horas) que antecedem e sucedem este pico, os quais registram valores menores que $200 \mathrm{nT}$. Caso essa condição se confirme, este prossegue como candidato à HILDCAA, e o contador da quantidade de picos 'y' avança para o próximo registro superior ou igual a $1.000 \mathrm{nT}$.

Depois de detectado a posição inicial e final do evento, é verificado o critério (1). O intervalo deve conter o mínimo de 2.880 pontos (dois dias). Este processo se repete até serem examinados todos os picos encontrados no conjunto total de dados para o ano de 1998. Para tal, utiliza-se a variável 'h' para representar a quantidade de pontos por candidato, que corresponde ao contador do intervalo de duração de cada candidato.

Para verificar se os possíveis candidatos não se encontram dentro da fase principal de tempestade geomagnética (critério 4), é necessário a inspeção visual nesse passo. Caso o candidato não registre valores menores que $-50 \mathrm{nT}$, o algoritmo automaticamente denomina o evento como HILDCAA/HILDCAA* pois atende a todos critérios necessários.

Em contrapartida, se o candidato registra valores inferiores à -50 nT será necessária a análise gráfica para o descarte da fase principal, e consequentemente, a certificação da duração temporal deste (critério 1). Primeiramente, o algoritmo plota os gráficos de cada evento, com os respectivos valores dos índices geomagnéticos - AE e SYM-H. Deve-se descartar o intervalo da fase principal anterior ao valor mínimo registrado pelo índice SYM-H e, a partir deste ponto verificar novamente os 3 critérios supracitados. Atendendo a estas condições, o Candidato é considerado HILDCAA, caso contrário, este é descartado.

Por fim, o algoritmo cria um arquivo com os índices AE com início e fim (dia, mês, ano, hora e minuto) de evento analisado. Este arquivo é utilizado para determinar o forçante interplanetário em questão responsável pelas variações geomagnéticas no índice AE (https://omniweb.gsfc.nasa.gov/), neste caso, se o forçante é CME e/ou CIR.

O mesmo passo a passo acima descrito é executado para a detecção de eventos HILDCAAs* , todavia com alteração na parte do algoritmo que se refere ao critério (3). Ao invés do algoritmo percorrer 120 pontos consecutivos que antecedem e sucedem a última posição do pico AE maior ou igual à $200 \mathrm{nT}$, ele percorre 240 pontos. Quanto ao restante, o método é idêntico ao relatado anteriormente, seguindo a mesma sequência de etapas.

\section{RESULTADOS E DISCUSSÃO}

Nesta seção, será mostrado exemplos de eventos identificados como HILDCAAs/HILDCAAs* ${ }^{*}$ e os Candidatos gerados como saída do algoritmo antes da inspeção visual. A partir da análise gráfica verifica-se por meio de um exame visual minucioso, quais Candidatos do algoritmo condizem com os critérios de 
HILDCAA/HILDCAA*

\subsection{Eventos de HILDCAAs}

Na Tabela 2 consta a quantidade de eventos HILDCAAs identificados e o horário de ocorrência dos mesmos, após a inspeção visual. Além disso, caso o evento seja consequência de algum mecanismo interplanetário responsável pelas variações geomagnéticas oriundas de tempestades - CIR ou ICME, estão registrados a data e horário do mecanismo, que por sua vez, constatam a correlação entre HSS/CIR e HILDCAA (causa-efeito). Os resultados descritos acima podem ser comparados aos resultados obtidos por Prestes et al. (2017) e Guarnieri (2006).

Tabela 2 - Eventos HILDCAAs no ano de 1998.

\begin{tabular}{ccccccc}
\hline Evento_Ano & Início & Término & Mec. & Início & Término & Autor \\
\hline 01_1998 & 24 abr, 17:10 & 27 abr, 16:46 & CIR & 23 abr, 17:29 & 25 abr, 00:00 & Os Autores \\
02_1998 & 23 jul, 06:11 & 25 jul, 12:32 & CIR & 22 jul, 12:00 & 23 jul, 20:00 & Os Autores \\
\hline 01_1998 & 22 jul, 20:56 & 25 jul, 12:32 & CIR & 22 jul, 12:00 & 23 jul, 20:00 & Prestes \\
\hline 01_1998 & 24 abr, 18:03 & 27 abr, 06:05 & CIR & 23 abr, 17:29 & 25 abr, 00:00 & Guarnieri \\
02_1998 & 22 jul, 21:09 & 25 jul, 12:25 & CIR & 22 jul, 12:00 & 23 jul, 20:00 & Guarnieri \\
\hline
\end{tabular}

Fonte: Os autores.

$\mathrm{Na}$ Figura 3, para cada evento mostram-se dois painéis. O painel superior corresponde ao índice $\mathrm{AE}$, no qual o eixo $\mathrm{X}$ horizontal representa a quantidade de pontos em minutos do evento para a verificação da duração deste, enquanto que o eixo vertical são os valores do índice AE em nano-Tesla (nT). Neste gráfico, a linha pontilhada na cor verde centrada em $1.000 \mathrm{nT}$ é utilizada como referência para identificarmos os picos de $\mathrm{AE}$ maiores ou iguais a $1.000 \mathrm{nT}$. Esta linha refere-se ao pico AE do critério (2) estabelecido por Tsurutani e Gonzalez (1987). Enquanto a linha pontilhada na cor preta faz menção ao critério (3) de HILDCAA/HILDCAA*, demarcando os valores de $200 \mathrm{nT}$. $O$ painel inferior apresenta o índice SYM-H, no qual o eixo $X$ representa a quantidade de pontos de minutos do evento, já o eixo vertical $Y$ marca os valores do índice SYM-H em nano-Tesla. No mesmo painel, há a existência de uma linha preta que é utilizada como referência para determinar os valores do índice SYM-H equivalentes à $-50 \mathrm{nT}$. Esta linha auxilia na inspeção visual, e refere-se ao critério (4). O valor "0" no eixo X corresponde ao início do evento HILDCAA* em ambos painéis. Nas Figuras 3(a) e 3(b), a HILDCAA está contida em uma HILDCAA*, portanto para verificação do início da HILDCAA usar seta vermelha como referência de início e fim. Entretanto nas Figuras 3(b) e 3(c), a linha vertical magenta refere-se ao final da fase principal de tempestade magnética, e possível descarte como será explicado a seguir. Nesses casos, a linha magenta indica o início dos eventos. Para maiores detalhes do início e fim de cada 
evento, ver Tabela 2.

Figura 3 - Eventos de HILDCAA/HILDCAA* detectados pelo algoritmo em Python em 1998.
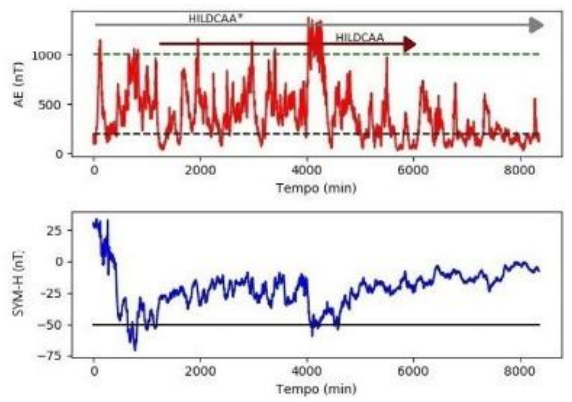

(a) Evento 01_1998 HILDCAA e 02*-1998 HILDCAA*.
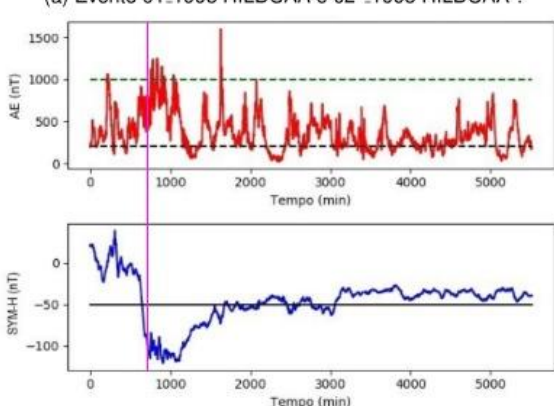

(c) Evento $01^{\star}-1998$ HILDCAA*.
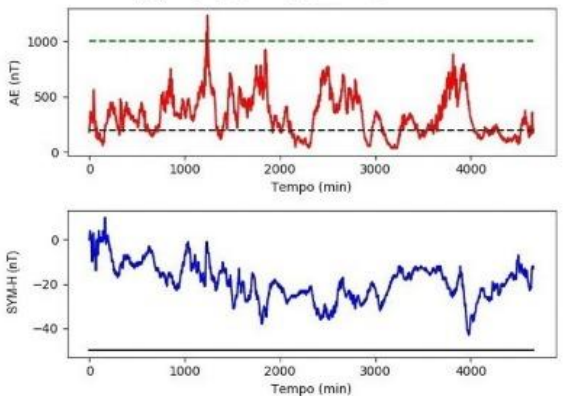

(e) Evento $04^{*}-1998$ HILDCAA* $^{*}$.
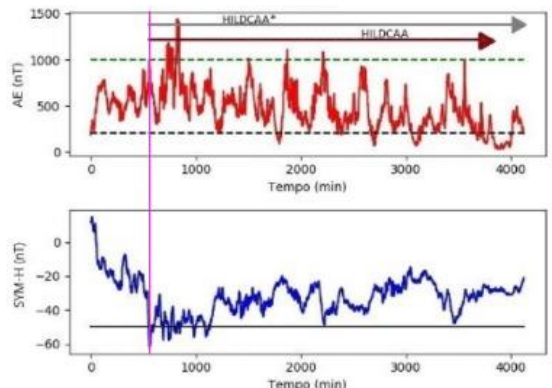

(b) Evento 02 -1998 HILDCAA e 06*_1998 HILDCAA*
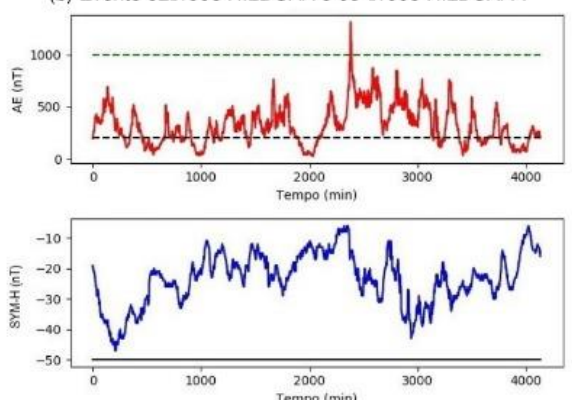

(d) Evento $03^{*}-1998$ HILDCAA $^{*}$.
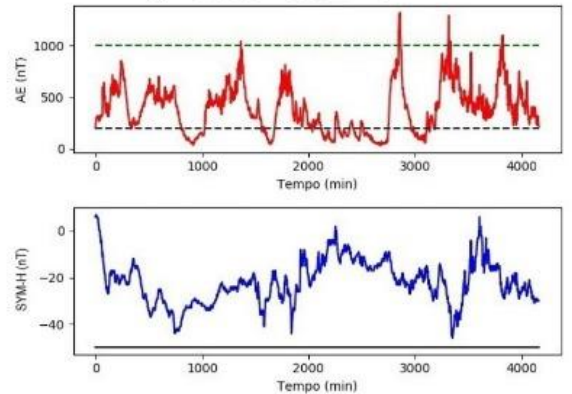

(f) Evento $05^{*}-1998$ HILDCAA* $^{*}$.
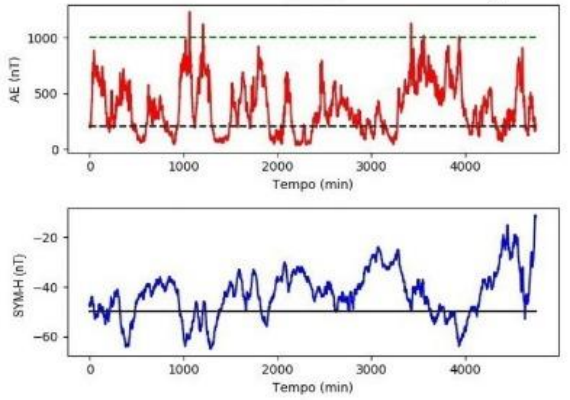

(g) Evento 07*-1998: Candidato a HILDCAA.

Fonte: Os autores.

A Figura 3(a) retrata nosso primeiro evento 01_1998 descrito na Tabela 2. Percebe-se que este evento ocorreu durante a fase de recuperação da tempestade geomagnética. Apesar de apresentar uma flutuação considerável do índice SYM-H, esta corresponde à uma injeção de energia associada a HSSs/CIRs, e, portanto, considerase como evento HILDCAA. Ademais, conforme os dados do Centro de Física Espacial 
da Universidade da Califórnia em Los Angeles (UCLA) houve episódio de mecanismo CIR durante este evento (http://wwwssc.igpp.ucla.edu/ jlan/ACE/Level3/SIR_List_from_Lan_Jian.pdf acessado em Janeiro, 2018).

Por outro lado, no segundo evento da Tabela 2 (02_1998) demonstrado na Figura 3(b), é necessário o descarte do intervalo condizente à fase principal da tempestade geomagnética, e consequentemente depois disto, o evento teve que ser reavaliado. Analisando o gráfico consoante ao índice SYM-H, iniciou-se a contagem do ponto do eixo $X$ referente a duração do evento, a partir do valor mínimo do índice SYM-H encontrado, ou seja, o ponto mínimo relativo a esse valor inferior à -50 nT (linha vertical magenta). Por conseguinte, verificou se o candidato possuía um pico de $1.000 \mathrm{nT}$ a partir desse ponto mínimo, como descreve a linha na cor vermelha, atendendo ao critério (2). Concluiu que partindo deste ponto, o evento ultrapassava 2.880 pontos, e, portanto, atende ao critério (1). Logo, é chamado de HILDCAA por meio da inspeção visual. Além disso, conforme os dados do Centro de Física Espacial da UCLA, houve episódio de mecanismo CIR durante este evento. Os resultados descritos acima podem ser comparados aos resultados obtidos por Prestes et al. (2017) e Guarnieri (2006), ver Tabela 2.

Verifica-se que a data tanto do início quanto do término do evento 01_1998 detectado no algoritmo em Python foi equivalente às datas do primeiro evento encontrado por Guarnieri (2006), todavia, este evento não foi identificado pelo algoritmo via MATLAB de Prestes et al. (2017). Apesar das datas terem sido compatíveis nos estudos supracitados, os horários de início e término deste evento foram distintos nos estudos realizados. Esse fato deve-se a flexibilização adotada pela metodologia deste artigo certamente foi diferente da utilizada por Guarnieri (2006). Percebe-se que esse evento teve início quase uma hora antes. Por outro lado, os horários de término foram consideravelmente díspares com variação de dez horas e quarenta e um minutos, o que se deve também a metodologia aplicada de cada autor.

Alia-se à discussão o segundo evento HILDCAA, que por sua vez foi identificado pelos três estudos em questão. Observa-se a consistência dessa ocorrência, todavia houve disparidade nos resultados registrados como constam nas tabelas. As datas iniciais do evento são iguais nos estudos de Prestes et al. (2017) e Guarnieri (2006), porém usando o algoritmo em Python foi registrado um dia depois o início deste, por conta do descarte da duração da fase principal da tempestade. Por outro lado, verificase que as datas finais dos eventos foram condizentes. Além disso, os horários de início também foram similares, visto que a diferença da hora inicial de Prestes et al. (2017) para Guarnieri (2006) foram de exatos 13 minutos, ver Tabela 2. Já os horários finais desse evento HILDCAA foram equivalentes na análise dos gráficos disponibilizados tanto em MATLAB quanto em Python, e estes se estenderam sete minutos a mais que o horário detectado por Guarnieri (2006). Ademais, assim como estabelecido nesse artigo o evento 02_1998 está associado com o mecanismo CIR. 


\subsection{Eventos de HILDCAAs*}

Do mesmo modo, aplicou-se os mesmos passos para encontrar as HILDCAAs*. A Tabela 3 consigna os eventos que foram denominamos de HILDCAA*, com base na flexibilização do critério (3) conforme estabelecido por Prestes et al. (2017). Dessa forma, têm-se as datas e os horários dos eventos, e as datas e os horários dos mecanismos físicos (CIR ou CME) associados aos eventos HILDCAAs*.

Tabela 3 - Eventos HILDCAAs* no ano de 1998.

\begin{tabular}{|c|c|c|c|c|c|c|}
\hline Evento_Ano & Início & Término & Mec. & Início & Término & Autor \\
\hline $01^{*} \_1998$ & 10 mar, 18:01 & 14 mar, 01:25 & CIR & 9 mar, 21:00 & 11 mar, 04:00 & Os autores \\
\hline 02*_1998 & $24 \mathrm{abr}, 07: 25$ & 29 abr, 13:44 & CIR & $23 \mathrm{abr}, 17: 29$ & $25 \mathrm{abr}, 00: 00$ & Os autores \\
\hline $03^{*}$ _1998 & 10 mai, 05:31 & 13 mai, 02:24 & - & - & - & Os autores \\
\hline $04^{*}$ 1998 & 20 jun, 03:15 & 23 jun, 08:50 & CIR & 18 jun, 18:00 & 20 jun, 12:00 & Os autores \\
\hline $05^{*}{ }_{1} 1998$ & 03 jul, 19:58 & 06 jul, 17:20 & CIR & 04 jul, 18:00 & 06 jul, 18:00 & Os autores \\
\hline $06^{*} \_1998$ & 23 jul, 06:14 & 25 jul, 17:36 & CIR & 22 jul, 12:00 & 23 jul, 20:00 & Os autores \\
\hline $07^{*} \_1998$ & 28 ago, $22: 44$ & 01 set, 05:54 & CIR & 26 ago, 06:40 & $28 \mathrm{abr}, 01: 00$ & Os autores \\
\hline $01^{*}$ _1998 & 24 abr, 11:59 & $26 \mathrm{abr}, 13: 00$ & CIR & 23 abr, 17:29 & $25 \mathrm{abr}, 00: 00$ & Prestes \\
\hline $02^{*} \_1998$ & 10 mai, 05:32 & 13 mai, 02:24 & - & - & - & Prestes \\
\hline $03^{*} \_1998$ & 20 jun, 03:16 & 23 jun, 08:50 & CIR & 18 jun, 18:00 & 20 jun, 12:00 & Prestes \\
\hline $04^{*}$ 1998 & 03 jul, 19:59 & 06 jul, 17:20 & CIR & 04 jul, 18:00 & 06 jul, 18:00 & Prestes \\
\hline 05*_1998 & 22 jul, 20:56 & 25 jul, 17:36 & CIR & 22 jul, 12:00 & 23 jul, 20:00 & Prestes \\
\hline
\end{tabular}

Fonte: Os autores.

Algumas HILDCAA* no ano de 1998 foram considerados Candidatos de acordo com o algoritmo, já que possuíam valores do índice SYM-H menores que $-50 \mathrm{nT}$. Dessa forma, como os eventos não podem acontecer durante a fase principal da tempestade, detecta-se o ponto mínimo do índice SYM-H em cada gráfico. Por conseguinte, descarta-se todo intervalo da fase principal da tempestade geomagnética a partir deste ponto mínimo do índice SYM-H (ver linha vertical magenta). Baseado no novo início determinado para o evento, verifica se os quatro critérios de HILDCAA* são atendidos. Sob essas circunstâncias, tem-se o evento 1 (apresentado na Figura 3(c)).

Outra peculiaridade é a HILDCAA contida em uma HILDCAA*, estas casualidades são ilustradas nas Figuras 3(a) e 3(b) que equivalem aos eventos: 02*_1998 e 06*_1998 da Tabela 3. As Figuras 3(d)-3(g) mostram os demais eventos descritos na Tabela 3.

Note-se que o evento 7 da Tabela 3 detectado pelo algoritmo em Python não precisa do descarte da fase principal de tempestade geomagnética, visto que apresenta comportamento de uma flutuação correspondente a uma injeção de energia prolongada associada à HSS/CIR (Figura 3(g)) relacionada a fase de recuperação de tempestade geomagnética.

Por outro lado, quando não há registros do índice SYM-H abaixo de $-50 \mathrm{nT}$, o evento certamente corresponde à HILDCAA, pois se encontra fora de uma fase principal de tempestade geomagnética, ou seja, atende também aos demais critérios de HILDCAA. Pode-se verificar esse tipo de eventos nas Figuras 3(d), (e) e (f). 
A Tabela 3 também apresenta os eventos estabelecidos por Prestes et al. (2017) como HILDCAA*, e, dessa forma, pode-se compará-los aos detectados no algoritmo em Python. Nota-se que a mesma análise não pode ser contrastada com os estudos de Guarnieri (2006), já que a flexibilização HILDCAA* foi definida somente posteriormente por Prestes et al. (2017).

Primeiramente, verifica-se que no algoritmo via Python houve dois eventos a mais do que aqueles obtidos por Prestes et al. (2017), entre eles, 01*_1998 e 07*_1998 (ver Tabela 3). Isto se deve a inspeção visual. Por outro lado, os nossos demais eventos foram compatíveis com aqueles listados por Prestes et al. (2017). Verifica-se que o evento $02^{*}{ }_{-} 1998$ condiz com o evento $01^{*}{ }_{-} 1998$ de Prestes et al. (2017), apesar dos dois eventos relatarem durações distintas. Nosso evento correspondeu à um Candidato à HILDCAA que teve descarte da fase principal, o que pode explicar a disparidade também nos horários de término dos eventos. Por outro lado, os eventos 03*_1998, 04*_1998, e 05*_1998 foram compatíveis com aqueles relatados por Prestes et al. (2017). A única disparidade assinalada nestes casos foi quanto ao horário inicial, pois os eventos detectados pelo algoritmo em Python iniciaram-se apenas um minuto antes daqueles listados pelo algoritmo em MATLAB. Enquanto os horários de término foram condizentes, assim como as datas de início e fim.

Ademais, todos os eventos relatados tanto neste artigo quanto por Prestes et al. (2017) como HILDCAA* estão associados ao mecanismo CIR, exceto o nosso evento $03^{*} \_1998$ equivalente ao $02^{*} \_1998$ de Prestes et al. (2017).

\section{CONCLUSÕES}

De acordo com os dados obtidos pelo algoritmo em Python, os eventos encontrados foram compatíveis com aqueles tabelados por Prestes et al. (2017) e por Guarnieri (2006). Foi verificado que as datas e os horários dos eventos correspondiam com relação a grande maioria dos eventos. Algumas ocorrências identificadas pelo Python apresentaram apenas uma ínfima disparidade com relação ao horário de início do evento. Esta diferença pode ser justificada por conta do algoritmo em Python não eliminar os intervalos da fase principal, sendo esta eliminada pela inspeção visual em alguns candidatos. Por ser manual, pode justificar algumas distinções dos nossos resultados com aqueles encontrados por Prestes et al. (2017) e por Guarnieri (2006).

Alia-se ao assunto os eventos detectados como HILDCAAs*, verifica-se que dentre os resultados estabelecidos aqui com aqueles registrados por Prestes et al. (2017), tiveram três eventos equivalentes, apresentando apenas um adiantamento de um minuto para o início dos eventos. Verifica-se também que houve um evento HILDCAA* condizente no estudo realizado aqui com a pesquisa desenvolvida por Prestes et al. (2017), porém com uma diferença de nove horas e dezoito minutos quanto ao início do evento. Ademais, percebe-se que houve novamente maior número de eventos, com exatamente o acréscimo de dois eventos, por conta da flexibilização 
adotada na nossa análise visual.

Todavia, o algoritmo desenvolvido aqui ainda não é autossuficiente, uma vez que, é necessário um exame minucioso dos candidatos para não haver descartes inapropriados, já que o algoritmo executa apenas três dos quatro critérios automaticamente que definem HILDCAA/HILDCAA*. Dessa forma, objetiva-se prosseguir os estudos de HILDCAAs/HILDCAAs* nos anos subsequentes de 1998, aprimorando o algoritmo, e, se possível, torná-lo autônomo para avaliação de todos os critérios. Busca em um futuro próximo, acrescentar ao algoritmo a detecção do ponto mínimo identificado na inspeção visual para alguns eventos, eliminando essa etapa manual, e, consequentemente, tornando ainda mais preciso os resultados descobertos.

\section{AGRADECIMENTOS}

Ao Conselho de Desenvolvimento Científico e Tecnológico (CNPq) pelo apoio financeiro (processo: 129109/2017-7) e à Fundação de Amparo à Pesquisa de São Paulo (FAPESP) pelo apoio financeiro (processo: 2018/02692-0).

\section{REFERÊNCIAS}

CHAPMAN, S.; FERRARO, V. C. A. A new theory of magnetic storms. Nature, v. 126, n. 3169, 1930.

CHAPMAN, S.; FERRARO, V. C. A. Terrestrial Magnetism, v. 36, n. 77, 1931.

CHAPMAN, S.; FERRARO, V. C. A. Terrestrial Magnetism, v. 38, n. 79, 1933.

GONZALEZ, W. D. et al. Solar wind-magnetosphere coupling during intense magnetic storms (1978-1979). Journal of Geophysical Research: Space Physics, v. 94, p. 8835-8851, 1989.

GONZALEZ, W. D. et al. What is a geomagnetic storm. Journal of Geophysical Research: Space Physics, v. 99, p. 5771-5792, 1994.

GUARNIERI, F. L. The Nature of Auroras During High-Intensity Long-Duration Continuous AE Activity (HILDCAA) Events: 1998 to 2001. In: MCPHERRON, R. et al. Recurrent Magnetic Storms: Corotating Solar Wind. [s.I.]: American Geophysical Union, 2006.

HAJRA, R. et al. Relativistic electron acceleration during high-intensity, long-duration, continuous AE activity (HILDCAA) events: Solar cycle phase dependences.

Geophysical Research Letters, 41, p. 1876-1881, 2014.

HAJRA, R. et al. Relativistic (e > 0.6, > 2.0, and > 4.0 MeV) Electron Acceleration at Geosynchronous Orbit during High-intensity, Long-duration, Continuous AE Activity (HILDCAA) Events. The Astrophysical Journal, v. 799, n. 39, 2015.

KLAUSNER, V. et al. Latitudinal and longitudinal behavior of the geomagnetic field during a disturbed period: A case study using wavelet techniques. Advances in Space Research, v. 58, p. 2148-2163, 2016a. 
KLAUSNER, V. et al. An alternative way to identify local geomagnetically quiet days: a case study using wavelet analysis. Annales Geophysicae, v. 34, p. 451-462, 2016b.

KLAUSNER, V. et al. Main patterns of the geomagnetic field: A case study using principal componente analysis. Physicae, v. 11, p. 1-11, 2015.

KLAUSNER, V. et al. Study of local regularities in solar wind data and ground magnetograms. Journal of Atmospheric and Solar-Terrestrial Physics, v. 112, p. 10-19, 2014.

KLAUSNER, V. et al. Characteristics of solar diurnal variations: A case study based on records from the ground magnetic station at Vassouras, Brazil. Journal of Atmospheric and Solar-Terrestrial Physics, v. 92, p. 124-136, 2013.

LAMIN I. C. P. et al. Criterio para identificacao de eventos hildcaa/hildcaa* baseado em Indices geomagneticos. In: ENCONTRO LATINO AMERICANO DE INICIAÇÃO CIENTÍFICA, 21, 2017. Anais... São José dos Campos: UNIVAP, 2017. Disponível em: http://www.inicepg.univap.br/cd/INIC_2017/anais/arquivos/RE_0554_0207_01.pdf. Acesso em: 01 mar. 2020.

PRESTES, A. et al. Statistical analysis of solar wind parameters and geomagnetic indices during HILDCAA/HILDCAA* occurrences between 1998 and 2007. Advances in Space Research, v. 60, p.1850-1865, 2017a.

PRESTES, A.; KLAUSNER, V.; GONZALEZ, A. O. High-intensity, long-duration, continuous AE activity events associated with Alfvénic fluctuations in 2003. Annales Geophysicae, v. 35. p. 1231-1240, 2017.

RATCLIFFE, J. A. An introduction to ionosphere and magnetosphere. São Paulo: Cambridge University Press, 1972.

SOUZA, A. M. et al. A study on the main periodicities in interplanetary magnetic field $\mathrm{Bz}$ component and geomagnetic AE index during HILDCAA events using wavelet analysis. Journal of Atmospheric and Solar-Terrestrial Physics, v. 149, p. 81-86, 2016.

TSURUTANI, B. T.; GONZALEZ, W. D. The cause of high-intensity long-duration continuous AE activity (HILDCAAS) - Interplanetary Alfven wave trains. Planetary and Space Science, v. 35, p. 405-412, 1987. 